\title{
Fabrication, structure and mechanical properties of indium nanopillars
}

\author{
Gyuhyon Lee , Ju-Young Kim, Arief Suriadi Budiman, Nobumichi Tamura, Martin Kunz , Kai \\ Chen, MichaelJ. Burek ${ }^{a}$, JuliaR. Greer, TingY. Tsui ${ }^{\text {a.* }}$ \\ ${ }^{a}$ Waterloo Institute of Nanotechnology, University of Waterloo, 200 University Ave. W., Waterloo, ON, Canada N2L 3G1 Division of Engineering \\ and Applied Science, California Institute of Technology, 1200 E. California Blvd, Pasadena, CA 91125, USA Center for Integrated \\ Nanotechnologies (CINT), Los Alamos National Laboratory (LANL), Los Alamos, NM 87545, USA Advanced Light Source (ALS), Lawrence \\ Berkeley National Laboratory (LBNL), 1 Cyclotron Road, Berkeley, CA 94720, USA
}

Abstract

Solid and hollow cylindrical indium pillars with nanoscale diameters were prepared using electron beam lithography followed by the electroplating fabrication method. The microstructure of the solid-core indium pillars was characterized by scanning micro-X-ray diffraction, which shows that the indium pillars were annealed at room temperature with very few dislocations remaining in the samples. The mechanical properties of the solid pillars were characterized using a uniaxial microcompression technique, which demonstrated that the engineering yield stress is 9 times greater than bulk and is $1 / 28$ of the indium shear modulus, suggesting that the attained stresses are close to theoretical strength. Microcompression of hollow indium nanopillars showed evidence of brittle fracture. This may suggest that the failure mode for one of the most ductile metals can become brittle when the feature size is sufficiently small.

Keywords: Plastic deformation; X-ray diffraction; Compression test; Electroplating; Yield phenomena

\section{Introduction}

With the recent progress in small-scale fabrication techniques, it is now possible to readily manufacture structures with a minimum feature size at the nanometer scale. In the integrated circuit industry, high-performance transistors with critical dimensions as small as $20 \mathrm{~nm}$ have been successfully produced. However, the mechanical behavior of these nanoscale structures has not been thoroughly understood. The majority of research conducted in this area has focused on metals with cubic structures, i.e. nickel [1-3], gold [4-6], copper [7], molybdenum [8-10] and niobium [11]. The results of all these studies show that the uniaxial compressive yield strength of crystalline cubic metals increases with reduced sample sizes [12]. Uchic and Dimiduk [2] developed focused ion beam (FIB) techniques to fabricate pure nickel and nickel alloy pillars with a diameter as small as $5 \mathrm{~lm}$. Their compression data revealed that the yield strength of small pillars can be 100 times greater than the bulk values. Greer et al. [4] and Volkert et al. [13] show similar size effects with the compression yield strength of FIB-fabricated solid and porous gold pillars, respectively. Recently, the yield strength of body-centered cubic (bcc) Mo-alloy single crystal pillars fabricated by a solid solution directional precipitation methods was examined by Bei et al. [14]. This pillar fabrication technique removes the potential damage created by the FIB milling process and results in a pristine microstructure, with no initial defects [15]. They demonstrated that the Mo-alloy pillar flow stress approached the theoretical strength value, $1 / 25$ of shear modulus. In contrast to the earlier works, no size-dependent hardening was observed in these Mo-alloy pillars prepared by a FIB-less technique most likely due to the pristine initial microstructure in these pillars. These findings suggest that both the initial microstructure, i.e. dislocation density, as well as the size, have to be taken into account when considering mechanical testing at small scales. 
In this work we investigated small-scale plastic deformation of indium nanopillars, a previously unstudied material and crystal structure. This material has a tetragonal crystalline structure and a low melting temperature of

156 0C. Indium and its compounds have many important applications in the field of nanotechnology. For example, it has been demonstrated that indium phosphide (InP) and indium arsenide-phosphide (InAsP) nanowires can be used as highly polarized photoluminescence and infrared photo-detection devices [16,17]. Vertical field-effect transistors have also been constructed with indium oxide $\left(\mathrm{In}_{2} \mathrm{O}_{3}\right)$ nanowires [18]. Therefore, understanding the mechanical properties of nanometer-scale indium structures may have a significant impact on the commercial success of these novel devices.

The low melting temperature of indium allows it to undergo room temperature annealing, which helps to reduce or eliminate fabrication-induced stresses, defects, and dislocations. In addition, the small as-deposited indium grains grow into large crystals at ambient conditions. Two different sample geometries were studied - solid core and hollow pillars. The deformation mode of hollow pillars is particularly interesting because of the large surface area to volume ratio. In these samples the dislocations should glide to the surface much more readily than in the solid core pillars with the same diameter due to the significant effects of the image stress. To avoid potential ion damage caused by the FIB milling technique, our indium pillars were fabricated via an electroplating method.

\section{Procedure}

Fig. 1 illustrates the process integration steps for the fabrication of nanometer-scale indium pillars. These pillars were fabricated on silicon substrates coated with electron beam evaporated chromium/gold bi-layer. A gold seed layer was selected as the cathode in subsequent electroplating steps while the chromium film was used to promote adhesion between gold and silicon substrate. The thickness of these layers was deemed non-critical, but generally maintained at 50-100 nm for the gold layer and 20-50 nm for the adhesion layer. Following deposition of the seed layer, polymethylmethacrylate (PMMA) diluted in anisole (supplied by Microchem Corp.) was spun on the substrates and then cured at $1800 \mathrm{C}$ for $3 \mathrm{~min}$ to form the final resist layer. These PMMA thin films were then patterned by using the electron beam lithography techniques to produce holes in the resist with the diameter of $400 \mathrm{~nm}$. The resists were exposed with a $30 \mathrm{kV}$ electron beam at dosages of $1250 \mathrm{lCcm}^{-2}$ and developed using a 1:3 mixture of methylisobutylketone and isopropyl alcohol (IPA). After the PMMA templates were produced, samples were separated into two different groups. One group underwent a $15 \mathrm{~s}$ oxygen plasma descum process while the other did not. This descum process was applied to reduce pillar tapering caused by electron beam lithography and to promote homogenous electroplating. This descum process was performed with a Trion Phantom Reactive Ion Etcher (RIE) system operated at room temperature, with a chamber pressure of $102 \mathrm{~m}$ Torr, plasma power of 20 $\mathrm{W}$, and oxygen flow rate of $40 \mathrm{sccm}$. Results from a separate descum experiment on a $1.5 \mathrm{~lm}$ thick blanket PMMAthin film showed the etch rate under these conditions is $1.67 \mathrm{~nm} \mathrm{~s}^{-1}$.

After patterning the PMMA films, indium was electroplated into the template structures by using a two electrode configuration system and a commercially available indium sulfamate solution (purchased from Indium Corporation of America). This sulfamate bath was maintained at ambient temperature and mechanically stirred during the deposition process. High-purityindium metal slab was used as a soluble anode while the conductive gold seed layer underneath the patterned PMMA resist acted as the cathode. The plating process began with a $3 \mathrm{~s}$ current pulse of $11.4 \mathrm{~mA} \mathrm{~cm}^{-2}$ and then followed by galvanostatic electroplating at $1.14 \mathrm{~mA} \mathrm{~cm}^{-2}$. The initial high current step was used to produce a thin indium seed layer which promoted homogenous filling of the patterned features and increased the final yield of indium pillars. Scanning electron microscopy (SEM) inspections show that greater than $50 \%$ of the patterned features were filled with indium metal when processed with the procedure described above. To achieve the pillar height of $800 \mathrm{~nm}$, the deposition time for the virgin and plasma-treated samples were set at 510 and $620 \mathrm{~s}$ respectively. After plating, specimens were rinsed in de-ionized water and the PMMA was removed using a commercial photoresist stripper (Microchemicals GmbH AZKwik Strip)at room temperature for at least $18 \mathrm{~h}$.

Uniaxial compression experiments of the indium pillars were conducted using Agilent G200 nanoindenter with a diamond flat punch approximately $10 \mathrm{~lm}$ in diameter. Since indium is a viscoelastic material, the yield strength of material is highly sensitive to the strain rate [19].To maintain a constant strain rate, all compression tests were performed with a constant nominal displacement rate of $2 \mathrm{nms}$ and a maximum displacement of $200 \mathrm{~nm}$.

\section{Results and discussion}

Fig. 2 shows a high-resolution scanning electron micrograph (SEM) of a $400 \mathrm{~nm}$ diameter indium pillar electrodeposited in a virgin PMMA mold without plasma treatment. It is clear from the image that the core of this metal pillar is solid. In contrast, PMMA molds which underwent the oxygen plasma pretreatment yielded hollow indium pillars after electroplating. Side and top-down SEM images of a $400 \mathrm{~nm}$ diameter indium pillar, which has a hollow core, are shown in Figs. 3 and 4, respectively. The top-down image (Fig.4) shows that the core of the metal column is a dark "hole", indicating that it is empty. Careful inspections of these pillars with different electron beam voltages, viewing angles, and other settings confirmed that the cores are indeed hollow from the indium seed layer upward while the sidewalls are continuous (images not shown here). Examinations of six other hollow indium pillars show that the wall thickness is 
thinner than $25 \mathrm{~nm}$. According to Schoeller and Cho [20], the native indium oxide thickness is 5nm at ambient conditions. Taking this into account, the actual thickness of indium metal side wall should be thinner than $15 \mathrm{~nm}$. The exact thickness is difficult to determine due to SEM resolution limit and the tendency for indium to melt or deform under extended electron beam exposures. Characterizing the sidewall by focused ion beam cross-section methods was also unsuccessful as the indium metal melts under ion beams even at the lowest beam current settings. However, it was observed that the intentionally overplated hollow pillars continue to grow into indium mushroom structures as the pillar height exceeds the PMMA mold thickness. This suggests that the sidewalls of the hollowed pillars are electrically conductive. A hollow pillar which has collapsed under the weight of its overplated mushroom structure during the PMMA removal process is shown in Fig.5. The circular nucleation site where the sidewall began to grow is clearly visible and has a diameter $400 \mathrm{~nm}$. Fig. 5 also indicates that the collapsed sidewall formed a thin indium blanket on the substrate surface. The mechanism to form hollow indium structures can be explained by an increased hydrophilic character of the PMMA molds caused by the oxygen plasma surface modification process. To quantify this, PMMA/distilled water contact angle tests were conducted before and after the plasma treatment. The results show that the contact angles reduced from 610 to 330 by this surface modification technique.

One of the challenges to characterize the crystalline structure of indium pillars is the localized joule heating effect. The rise in the sample temperature caused by the electron or ion beam exposure is conducive to the initiation of dislocation motion, defect annihilations, grain growth, deformation, and even melting. This eliminates the use of conventional microstructure characterization techniques, such as electron backscattered diffraction (EBSD), focused ion beam (FIB), and transmission electron microcopy (TEM). In order to avoid these artifacts, a non-destructive scanning micro-X-ray diffraction technique (uSXRD) was selected to characterize solid indium pillars. This method uses a focused polychromatic X-ray beam to obtain Laue diffraction patterns of indium pillars. These results can be used to determine the grain orientations, stresses and strains [21], and, more importantly, dislocation densities [22]. Micro-diffraction experiments were carried out by using a synchrotron radiation at the Beamline 12.3.2 Advanced Light Source (ALS) Synchrotron facility of the Lawrence Berkeley National Laboratory. Details of this white beam Laue technique have been described elsewhere [21-24]. Indium pillars prepared for this part of study were fabricated on a silicon substrate coated with 20 nm titanium adhesion layer and a $100 \mathrm{~nm}$ gold seed layer using the electroplating conditions described above. Initial high current density pulse was omitted for the X-ray diffraction specimens. Indium pillars examined by X-ray diffraction had solid cores with diameter of $250 \mathrm{~nm}$ and $1 \mathrm{~lm}$ tall. The pillars were again annealed at room temperature for approximately 30 days prior to the X-ray characterizations. Two distinct indexations of the Laue diffraction patterns were collected from the indium pillar shown in Fig.6a and indicate that the pillar consists of two large grains with out-of-plane orientations near h2010i and h019i, respectively. The diffraction spots belonging to indium crystals had similar intensities, suggesting that the grain sizes are comparable in size. Dislocation structures within these two crystals were determined by analyzing the shape of the Laue diffraction spots. Fig.6b and c shows the(215) and (0111) Laue diffraction spots of the grain with h2010i out-of-plane orientation, respectively. A circular intensity pattern surrounding these representative spots is observed, which indicates a fully annealed indium crystal with no excess initial dislocations of the same sign. Fig.6dande reveals the(118) and(0010) diffraction spots from the grain with h019iout-of-plane orientation, respectively. In contrast, these spots have a distinct streak intensity pattern. This suggests non-uniform plastic deformation and the presence of fabrication-induced geometry necessary dislocations (GNDs) [22]. By using the Cahn-Nye analysis [25,26] and a Laue spot streak length of 1.20, the GND density in this grain was estimated to be $3.35 \times 10^{9} \mathrm{~cm}^{-2}$, which translates to 4.2 immobile geometrically necessary dislocations in this pillar. The number of dislocations in the h019i oriented grain is calculated by multiplying the measured dislocation density by the area of interest with respect to the relevant crystal dimensions. The lack of dislocations in the h2010i oriented grain is consistent with the extended room temperature anneal ( 0.7 homologous temperature of indium) before the micro-Xray diffraction experiments. Even though at least four dislocations exist in the h019i oriented grain, we speculate that these dislocations are immobile for the following rea sons: (1) mobile dislocations are expected to annihilate at the free surfaces of the pillar during the extended annealing at 0.7 homologous temperature of indium, and (2) uniaxial compressive loading of the pillars (presented in the following section)suggests the plasticity observed is insen sitive to the remaining dislocations. It may be argued, however, that residual stresses in the pillar could stabilize mobile dislocations throughout the annealing step. In the future, the dislocation population in the pillar microstructure before and after compression will be investigated.

Nanometer-scale compression experiments were performed at room temperature on both solid and hollow pillars. These specimens were again annealed at room temperature for 30 days prior to the mechanical tests. A typical load-displacement(L-D)curve of solid indium pillar is revealed in Fig.7. This L-D curve consists of two distinct displacement bursts at compressive displacements near 30 $\mathrm{nm}$ and $130 \mathrm{~nm}$. Displacement data from these two events are plotted as a function of time in Figs. 8 and 9, respectively. They show two distinct deformation characteristics. Fig. 8 shows the low load burst actually consists of three independent "pop-in" events. The first one at $30 \mathrm{~nm}$ completed in $0.2 \mathrm{~s}$ while the indenter tip travelled $18 \mathrm{~nm}$. The other two events occurred within the next $3 \mathrm{~s}$ with combined displacement of $15 \mathrm{~nm}$. The indenter displacement remains constant in the following $18 \mathrm{~s}$. Due to the fragile nature of the hollow pillars, small sample size, and the low melting temperature of indium, it is extremely challenging to characterize them with great confidence. A technique is yet to be developed to analyze these hollow features. This type of "pop-in" displacement at small depths has been reported in gold, copper, molybdenum, molybdenum-alloy, and niobium pillar compression tests [4,6,7, 9-11,14,27]. It is often described as the incipient onset of plasticity where dislocations are nucleated within the pillars, allowing them to deform plastically. As the flat punch indenter continues to compress the indium pillar, the L-D curve(Fig.7)increases linearly until another displacement burst is observed near $130 \mathrm{~nm}$. Since the slope in this region is very steep and closely resembles to the unloading sti - 
ness, the displacement in this regime is most likely dominated by elastic deformation. Interestingly, the high load burst event at $130 \mathrm{~nm}$ has a di erent characteristic from those at small depths. It is preceded by a smooth transition of load-displacement curve prior to the burst rather than a sudden jump in displacement. This is possibly the onset of strain hardening, where the existing dislocations might be interacting with one another rather than nucleating. However, the amount of strain hardening is very small because it is immediately followed by a displacement burst and strain softening. The lack of work-hardening capacity in the indium pillars is consistent with its bulk property where recovery occurs readily at room temperature. Another distinction of this high load burst behavior is a smooth displacement motion rather than a "pop-in" deformation as illustrated in Fig.9. After the high load burst, Fig.7 shows the load continues to fall and proceeds into the unloading segment of the compression test. A selected group of indium pillar engineering stress-strain curves is plotted in Fig. 10 where the stress values are the ratios between the compression load and the pillar initial cross-sectional area. This figure shows that the "pop-in" events at low load and softening at high load are common phenomena.

As the X-ray diffraction results presented above describe, these indium pillars have been fully annealed at room temperature with very few pre-existing defects and immobile geometrically necessary dislocations. The results from six indium pillars with solid cores are summarized in Table1. The engineering yield stress attained from compression is defined as the flow stress associated with the first low load pop-in. The ultimate strength is the maximum stress value prior to the softening deformations. The average indium pillar yield stress and ultimate strength were determined to be $181 \pm 77 \mathrm{MPa}$ and $606 \pm 112 \mathrm{MPa}$, respectively. These values correspond to $1 / 28$ and $1 / 7$ of bulk indium shear modulus $(\mathrm{G}=4.35 \mathrm{GPa})$ [28], respectively, i.e. the nanoscale indium pillars indeed approach the theoretical strength of indium. The pillar yield strength is 9 times greater than the bulk value of $20 \mathrm{MPa}$. The bulk value was estimated by using the nanoindentation hardness values reported by Lucas and Oliver [19] under the same indentation strain rate conditions and the assumption that hardness is 3 times the yield strength [29]. Since the indium nanopillars yield near their theoretical strength, our assumption that the limited initial dislocations in the pillar microstructure are immobile is not unfounded. If the present dislocations were, in fact, mobile, they would dominate the plasticity of the pillar and result in a final yield stress significantly less than the theoretical strength. This demonstrates that nanometer-scale indium pillar can exhibit exceptional mechanical strength when the initial microstructure is nearly pristine.

The L-D curves of four different hollow pillars are plotted in Fig. 11. These curves illustrate typical deformation characteristics observed in the hollow indium pillars. Unlike the solid pillar L-D curves in Fig.7, the hollow pillar data do not exhibit any displacement bursts. Fig. 11 shows the curves have a fairly linear slope with virtually no plastic deformation prior to failure at compressive loads smaller than $15 \mathrm{IN}$. Typical SEM images of a failed hollow pillar are shown in Fig. 12. The figure shows that the sidewalls fractured with no significant bending or buckling, which is consistent with the idea that the failure is brittle in nature. The native indium oxide represents $40 \%$ of the volume in the case of the hollow indium pillars. As such, it is easy to attribute the brittle failure observed in these samples to the typical brittle failure exhibited by oxides. However, since the indium sidewall thickness is less than $15 \mathrm{~nm}$ and the distance where the dislocations travel to the surface is very short, we can speculate that it is unlikely that dislocations nucleated during deformation will reside in the metal. As such, these hollow samples will most likely not contain any mobile dislocations and result in ductile behavior characteristic of extended dislocation activity. Therefore, the mechanical failure of these structures will be dominated by the non-dislocation-driven deformation processes like grain boundary sliding and brittle fracture. This suggests that the failure mode for one of the most ductile metals can become brittle when the feature size is suffciently small.

\section{Conclusions}

In conclusion, we have demonstrated an effective method for the fabrication of solid and hollow indium nanopillars suitable for nanomechanical testing, as well as for other integration steps of nanoscale indium. Uniaxial compression tests of solid indium pillars reveal a dramatic increase in strength over that of bulk indium and approach the ideal strength of this metal. This is consistent with the X-ray micro-diffraction results which reveal a very small number of initial dislocations within the indium pillar microstructure. Microcompression of indium nanopillars with a hollow structure showed evidence of brittle fracture; this is also consistent with the escape of dislocations before the initiation of plasticity.

\section{Acknowledgements}

The authors gratefully acknowledge critical support and infrastructure provided for this work by the Kavli Nanoscience Institute at Caltech and the Western Nanofabrication Facility at the University of Western Ontario. T.Y. Tsui thanks Easo George, George M. Pharr, and Joost J. Vlassak for valuable discussions. His research projects are partially supported by Canadian NSERC Discovery and RTI Grants. TYT would like to thank ArashTajik for his assistance in this project. The Advanced Light Source is supported by the Director, Office of Science, Office of Basic Energy Sciences, Materials Sciences Division, of the US Department of Energy under Contract No. DE-AC0205CH11231 at Lawrence Berkeley National Laboratory and University of California, Berkeley, California. The move of the micro-diffraction program from ALS beamline 7.3.3 onto to the ALS superbend source 12.3.2 was enabled through the NSF Grant \#0416243. One of the authors (ASB) is supported by the Director, Los Alamos National Laboratory (LANL), under the Director's Postdoctoral Fellowship program (\#20090513PRD2).

References 
[1] Uchic MD, Dimiduk DM, Florando JN, Nix WD. Science 2004;305:986.

[2] Uchic MD, Dimiduk DM. Mater Sci Eng A 2005;400-401:268.

[3] Dimiduk DM, Uchic MD, Parthasarathy TA. Acta Mater 2005;53:4065.

[4] Greer JR, Oliver WC, Nix WD. Acta Mater 2005;53:1821.

[5] Volkert CA, Lilleodden ET. Philos Mag 2006;86:5567.

[6] Kiener D, Grosinger W, Dehm G, Pippan R. Acta Mater 2008;56:580.

[7] Kiener D, Motz C, Scho“ berl T, Jenko M, Dehm G. Adv Eng Mater 2006;8:1119.

[8] Brinckmann S, Kim J-Y, Greer JR. Phys Rev Lett 2008;100:155502.

[9] Kim J-Y, Greer JR. Appl Phys Lett 2008;93:101916.

[10] Kim J-Y, Greer JR. Acta Mater 2009;57:5245.

[11] Kim J-Y, Jang D, Greer JR. Scripta Mater 2009;61:300.

[12] Uchic MD, Shade PA, Dimiduk DM. Annu Rev Mater Res 2009;39:361.

[13] Volkert CA, Lilleodden ET, Kramer D, Weissmuller J. Appl Phys Lett 2006;89:061920.

[14] Bei H, Shim S, George EP, Miller MK, Herbert EG, Pharr GM. Scr Mater 2007;57:397.

[15] Kiener D, Motz C, Rester M, Jenko M, Dehm G. Mater Sci Eng: A 2007;459:262.

[16] Wang J, Gudiksen MS, Duan X, Cui Y, Lieber CM. Science 2001;293:1455.

[17] Pettersson H, Tragardh J, Persson AI, Landin L, Hessman D, Samuelson L. Nano Lett 2006;6:229.

[18] Nguyen P, Ng HT, Yamada T, Smith MK, Li J, Han J, et al. Nano Lett 2004;4:651.

[19] Lucas B, Oliver W. Metall Mater Trans A 1999;30:601.

[20] Schoeller H, Cho J. J Mater Res 2009;24:386.

[21] Tamura N, MacDowell AA, Spolenak R, Valek BC, Bravman JC, Brown WL, et al. J Synchrotron Radiat 2003;10:137.

[22] Budiman AS. Probing plasticity at small scales: from electromigration in interconnects to dislocation hardening processes in crystals. Stanford: Department of Materials Science and Engineering; 2008.

[23] Budiman AS, Nix WD, Tamura N, Valek BC, Gadre K, Maiz J, et al. Appl Phys Lett 2006;88:233515.

[24] Budiman AS, Han SM, Greer JR, Tamura N, Patel JR, Nix WD. Acta Mater 2008;56:602.

[25] Cahn RW. J Inst Metals 1949;76:121.

[26] Nye JF. Acta Metall 1953;1:153.

[27] Greer JR, Nix WD. Appl Phys A: Mater Sci Process 2005;80:1625.

[28] Ledbetter H, Sizova N, Kim S, Kobayashi H, Sgobba S, Parrini L. J Phys IV France 1996;06:C8.

[29] Hill R. The mathematical theory of plasticity. New York: Oxford University Press; 1971. 


\section{Silicon}

1. substrate

$\mathrm{Cr} / \mathrm{Au}$ Seed

Silicon

2. coating $\mathrm{Cr} / \mathrm{Au}$ seed

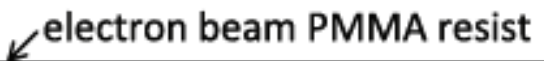

Silicon

3. PMMA resist coating

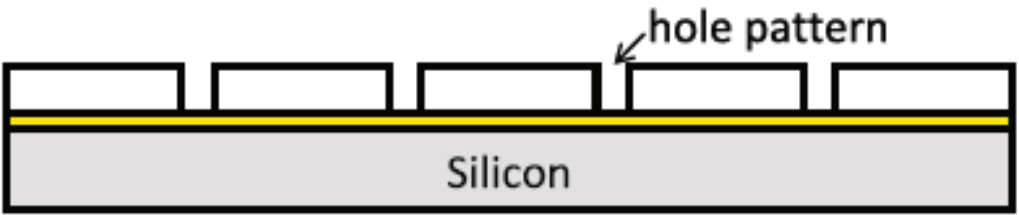

4. resist exposure and development

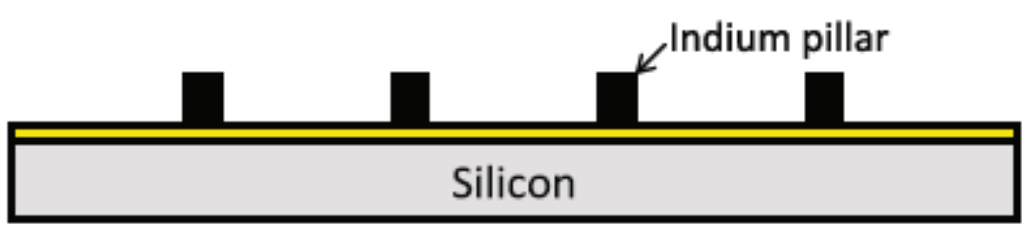

5. indium plating and resist removal

Fig. 1. Schematic illustration of the indium pillar fabrication process.

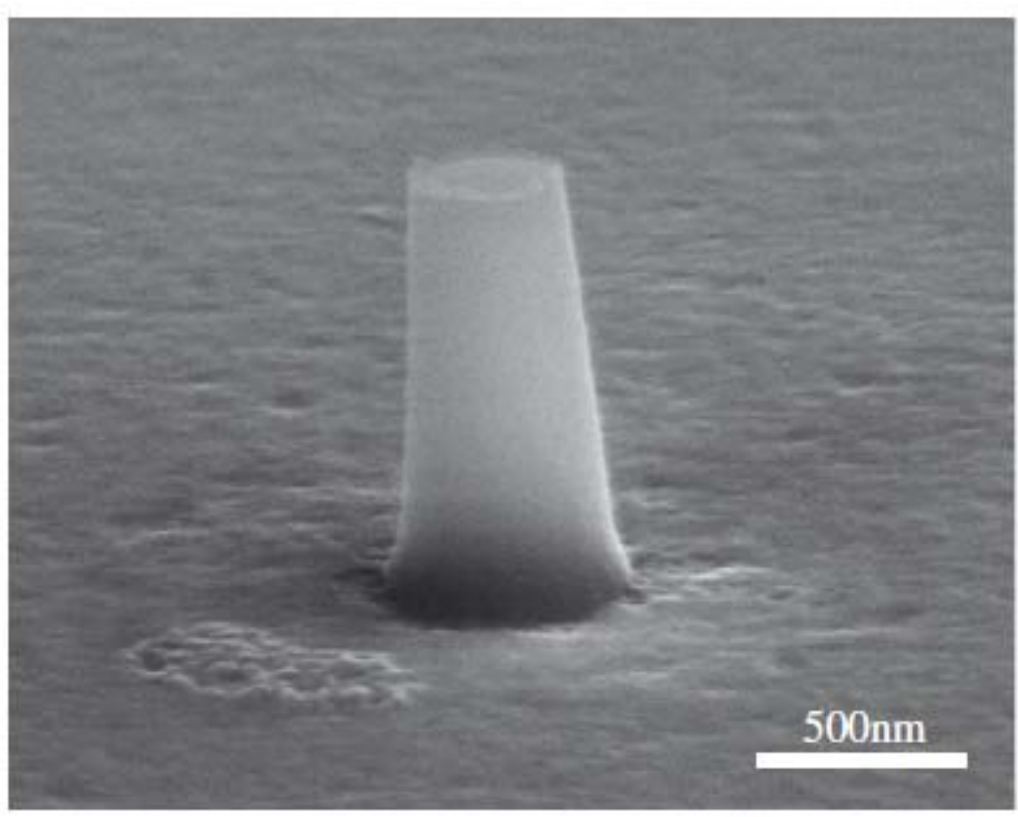

Fig. 2. SEM image of an indium pillar with solid core. 


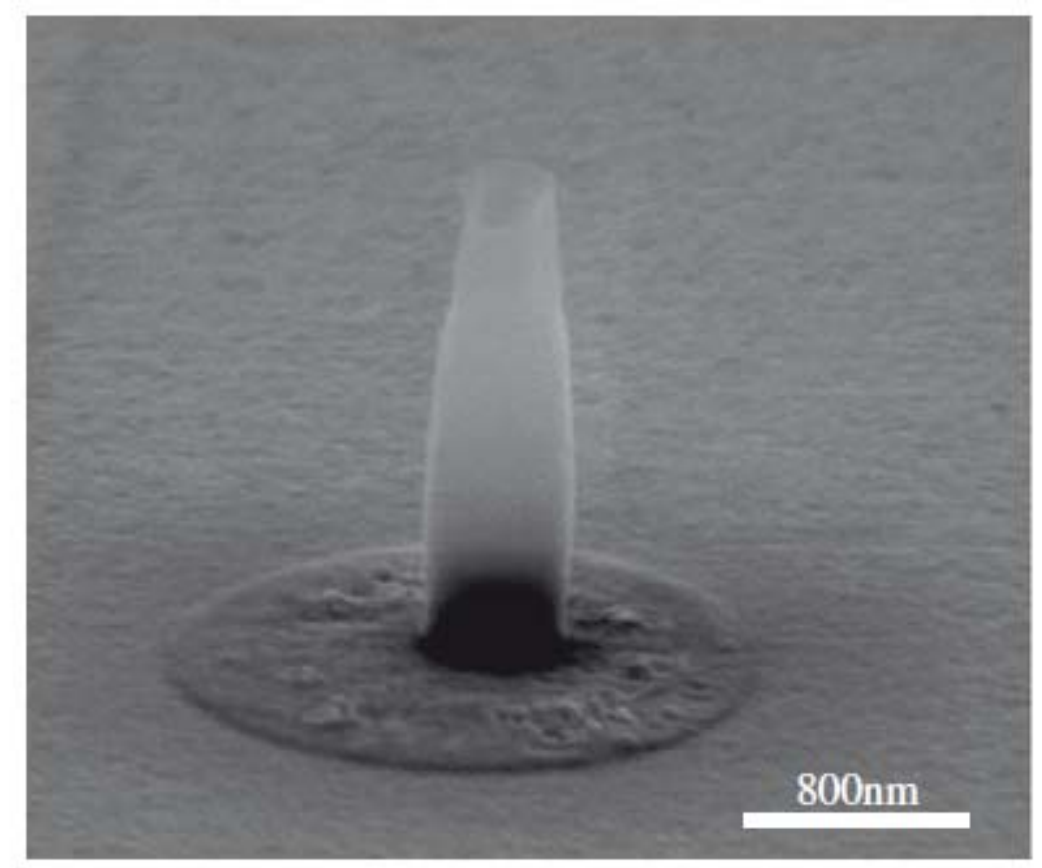

Fig. 3. SEM image of an indium with hollow core structure.

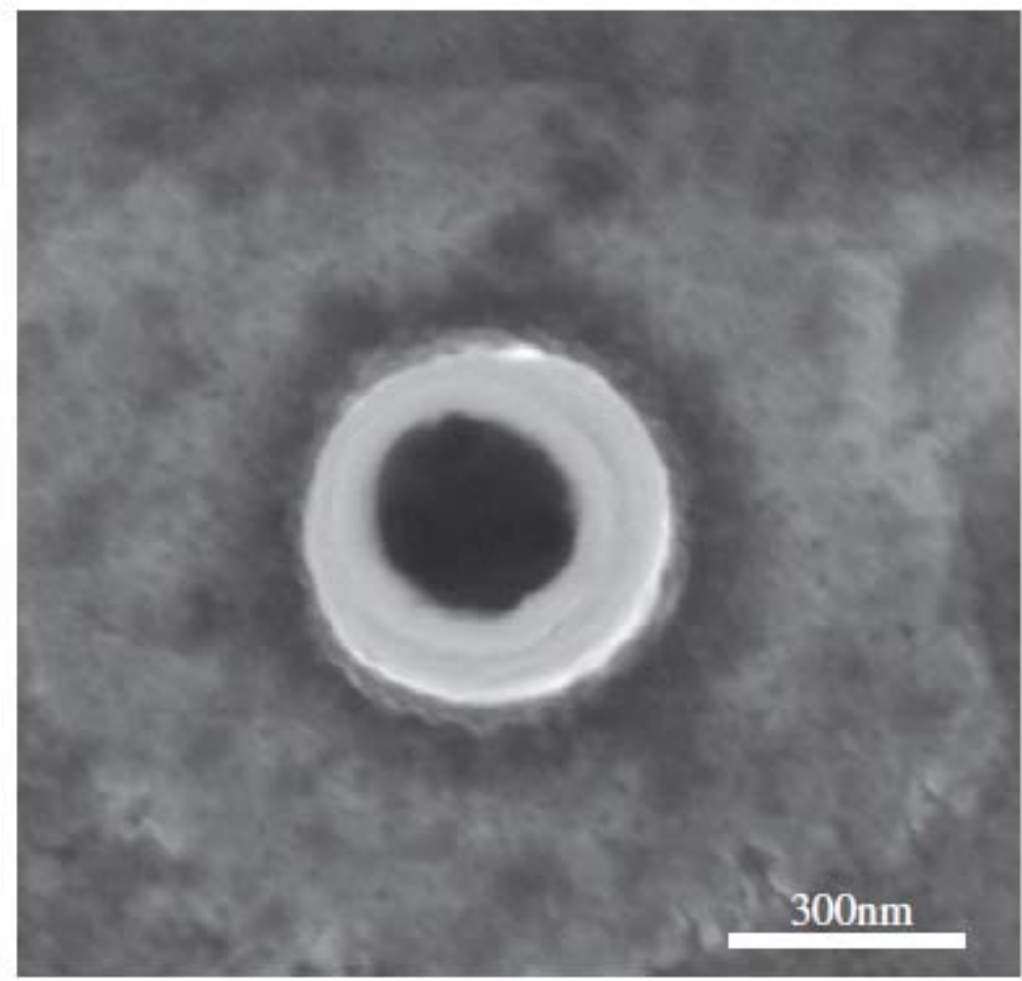

Fig. 4. Top-down SEM image of a hollow pillar. 


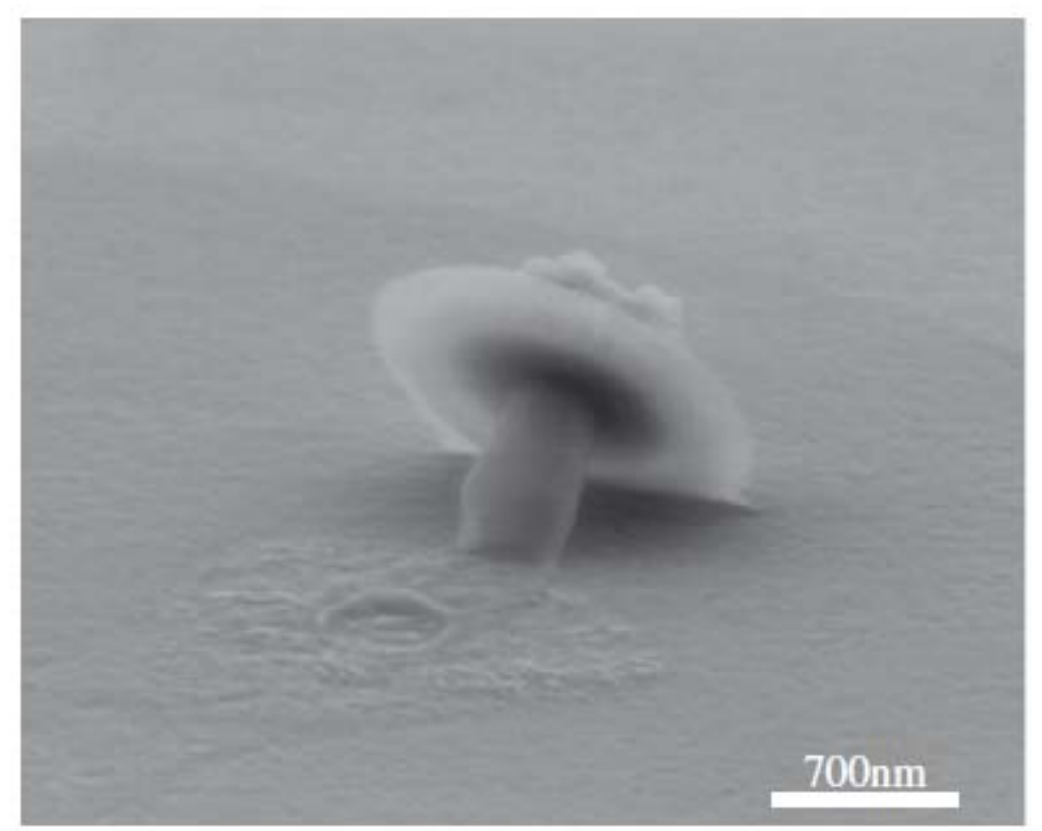

Fig. 5. Collapsed hollow pillar with the intentionally overplated mushroom top.

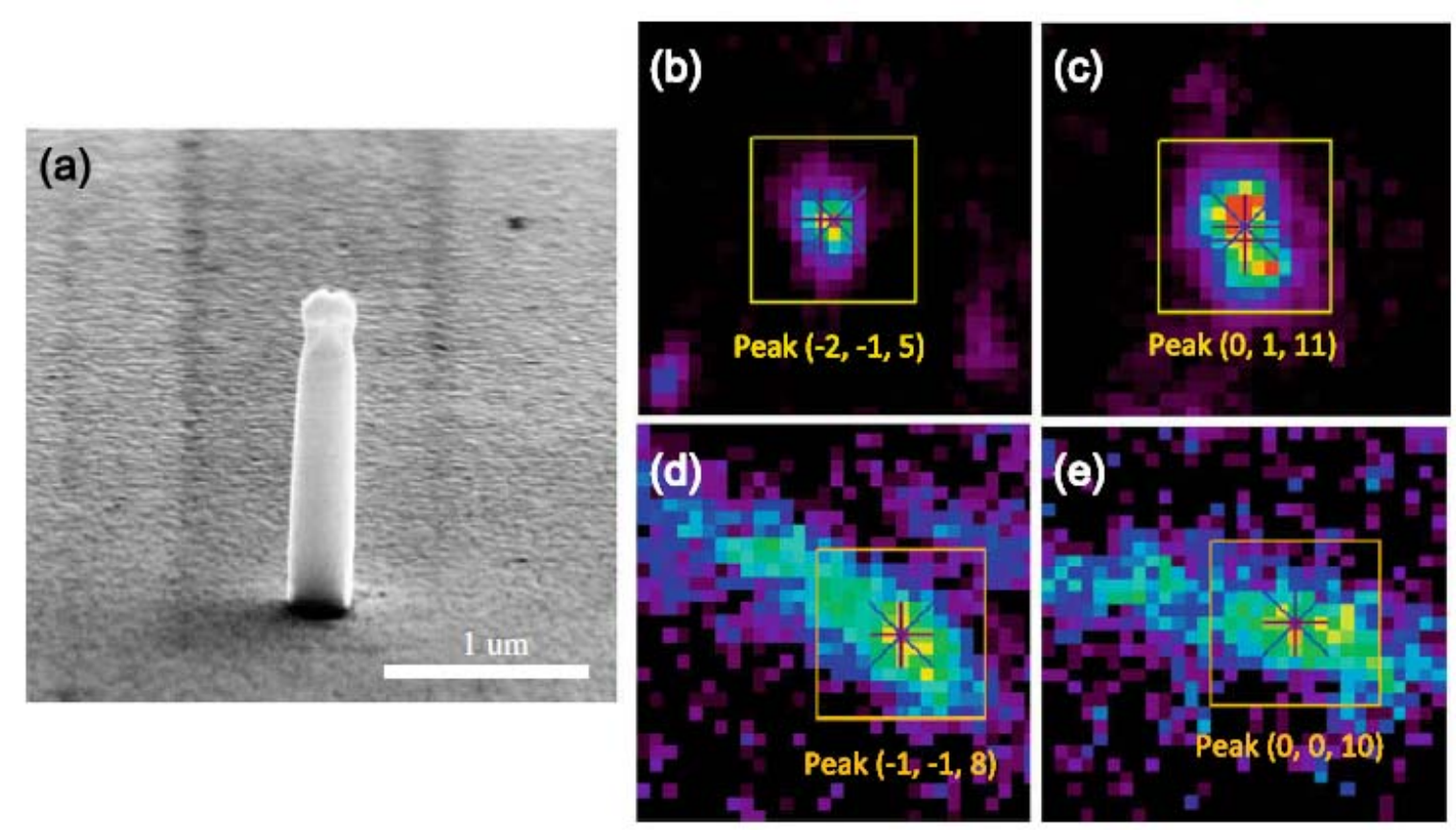

Fig. 6. (a) SEM image of an indium pillar characterized by scanning micro-X-ray diffraction. (b) The $(\overline{21} 5)$ peak taken from the Laue diffraction patterned generated by the indium pillar grain with $\langle\overline{2} 010\rangle$ out-of-plane orientation. (c) The $(0111)$ peak taken from the Laue diffraction patterned generated by the indium pillar grain with $\langle 2010\rangle$ out-of-plane orientation. (d) The (118) peak taken from the Laue diffraction patterned generated by the indium pillar grain with $\langle 0 \overline{1} 9\rangle$ out-of-plane orientation. (e) The $(0010)$ peak taken from the Laue diffraction patterned generated by the indium pillar grain with $\langle 0 \overline{1} 9\rangle$ out-of-plane orientation. 


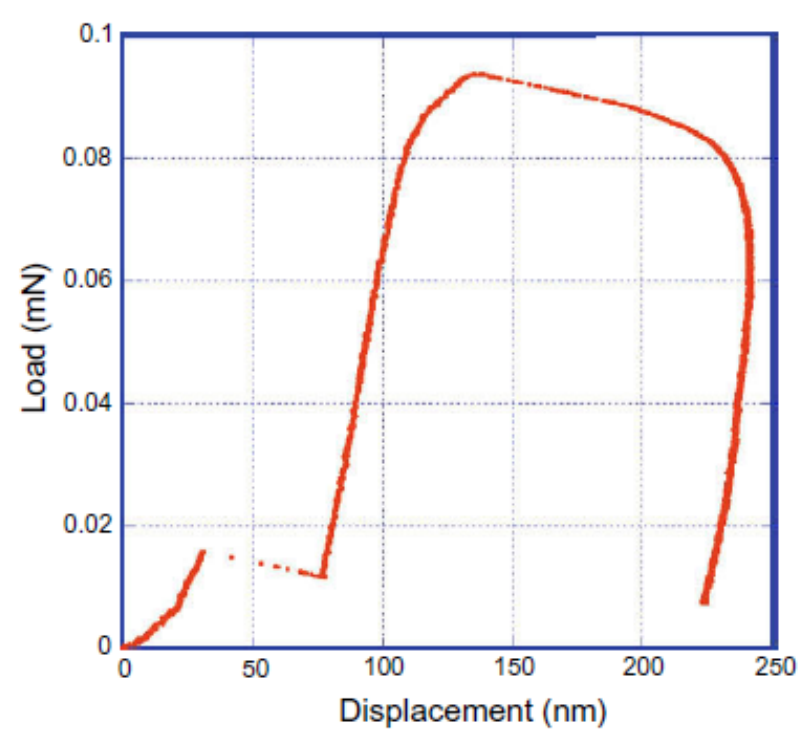

Fig. 7. Load-displacement curve of a solid indium pillar.

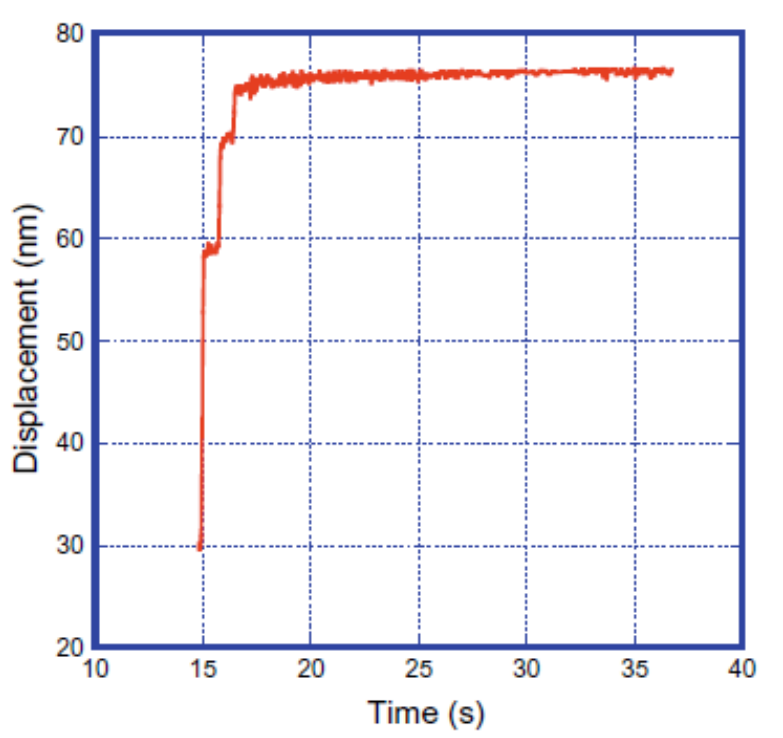

Fig. 8. Plot of low load-displacement burst as a function of time.

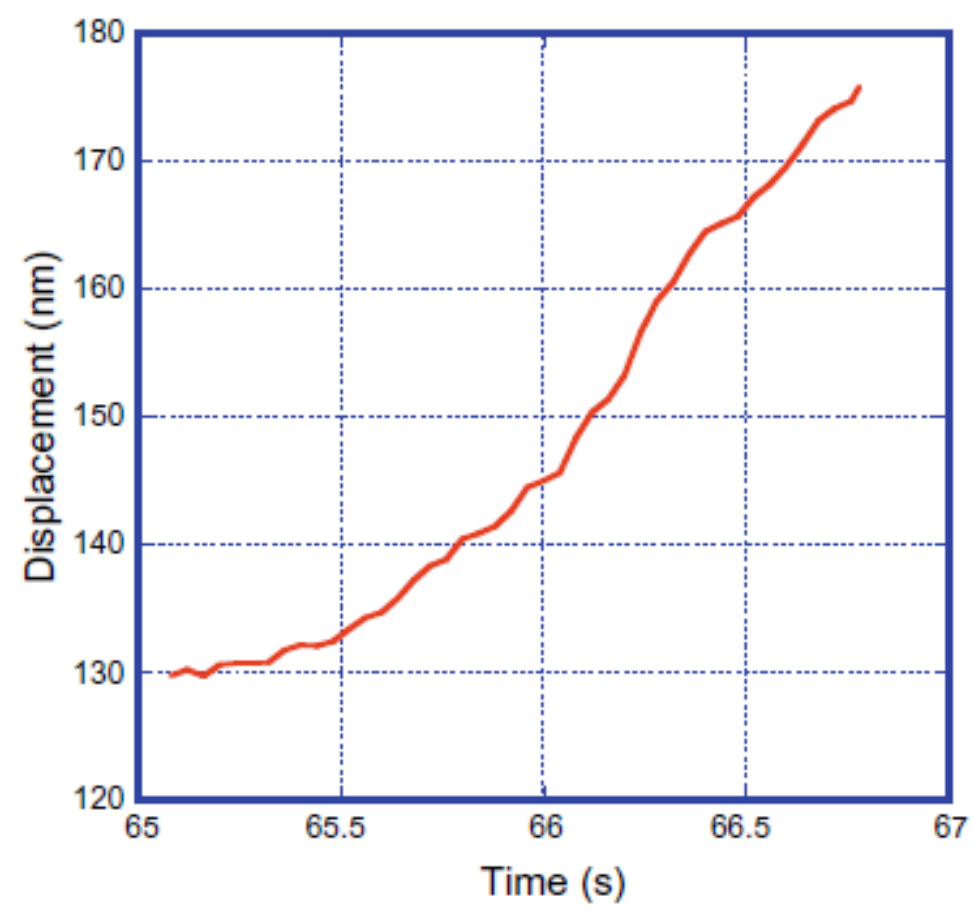

Fig. 9. Plot of low displacement burst as a function of time under high load conditions. 
Table 1

Engineering yield stress and ultimate strength of solid indium pillars.

\begin{tabular}{lllll}
\hline $\begin{array}{l}\text { Pillar diameter } \\
(\mathrm{nm})\end{array}$ & $\begin{array}{l}\text { Engineering yield } \\
\text { stress, } \sigma_{\mathrm{y}}(\mathrm{MPa})\end{array}$ & $\begin{array}{l}\text { Ultimate strength, } \\
\sigma_{\mathrm{u}}(\mathrm{MPa})\end{array}$ & $G / \sigma_{\mathrm{y}}$ & $G / \sigma_{\mathrm{u}}$ \\
\hline 373 & N/A & 473 & N/A & 9.2 \\
388 & 242 & 548 & 18.0 & 7.9 \\
381 & 147 & 716 & 29.6 & 6.1 \\
428 & 110 & 651 & 39.5 & 6.7 \\
370 & 283 & 739 & 15.4 & 5.9 \\
440 & 121 & 508 & 36.0 & 8.6 \\
Average & 181 & 606 & 28.0 & 7.0 \\
Std. dev. & 77 & 112 & 11.0 & 1.0 \\
\hline
\end{tabular}

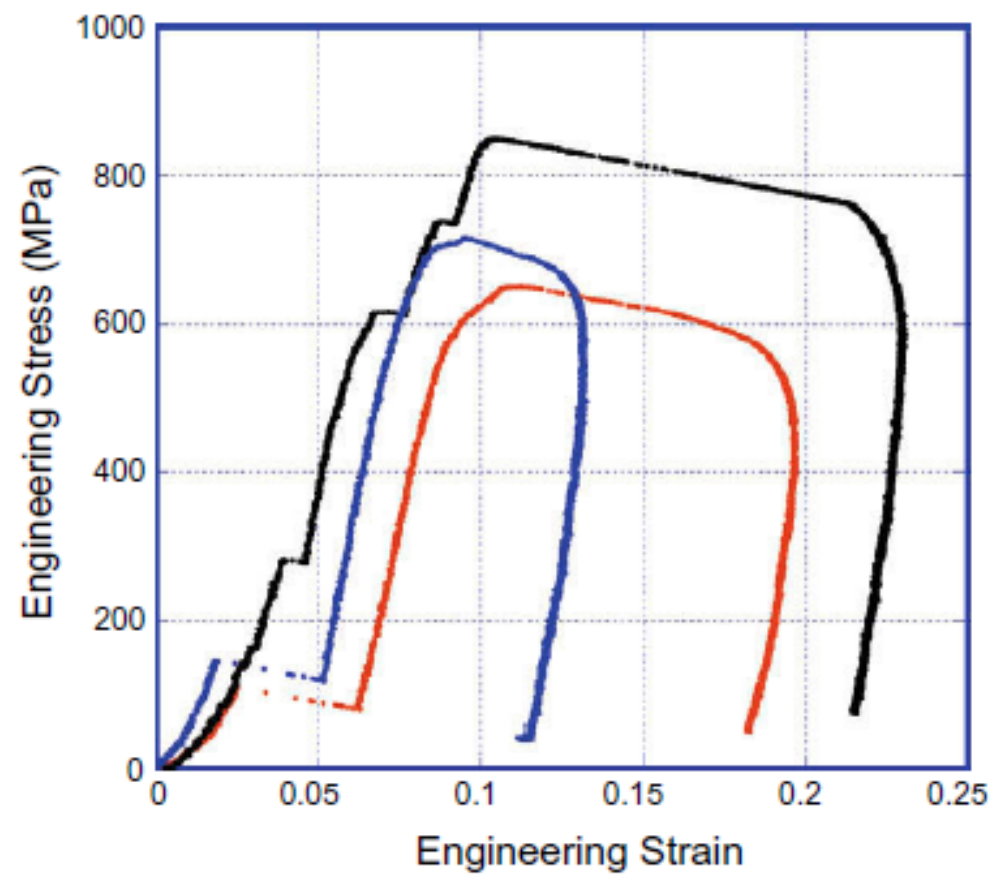

Fig. 10. Engineering stress-strain curves of solid indium pillars. 


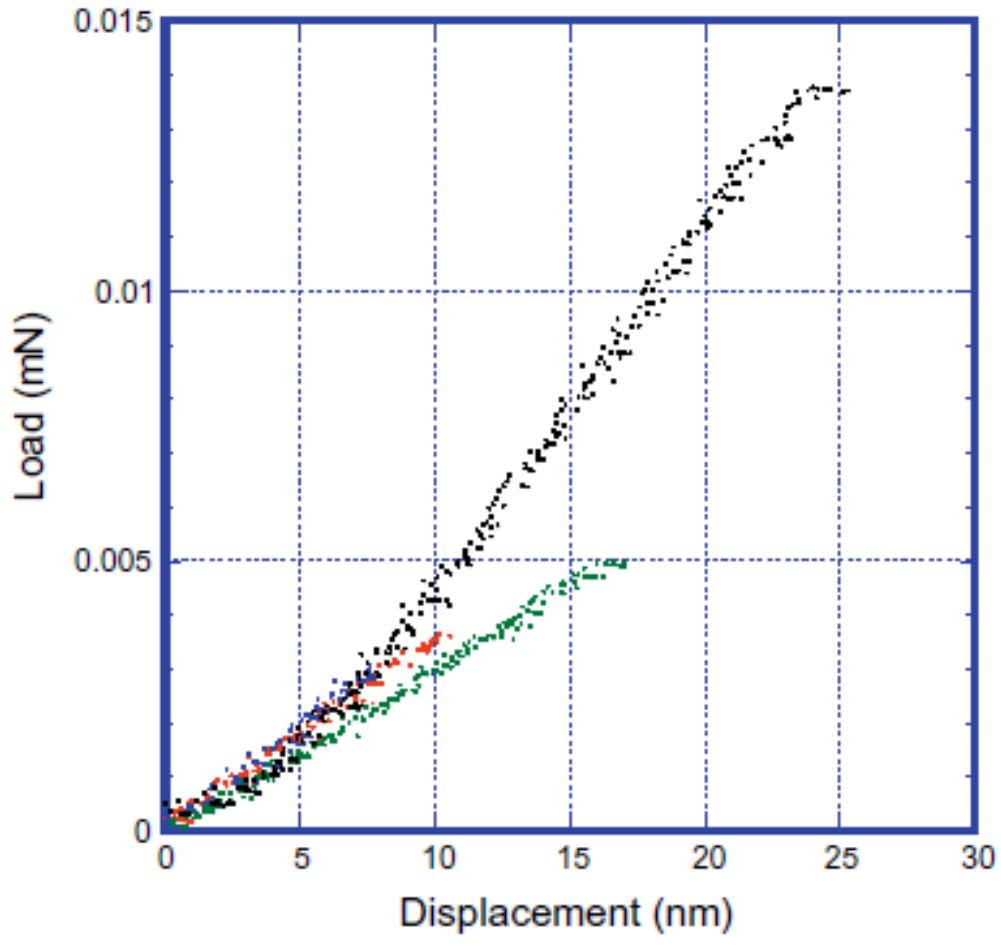

Fig. 11. Load-displacement curve of hollow indium pillars.
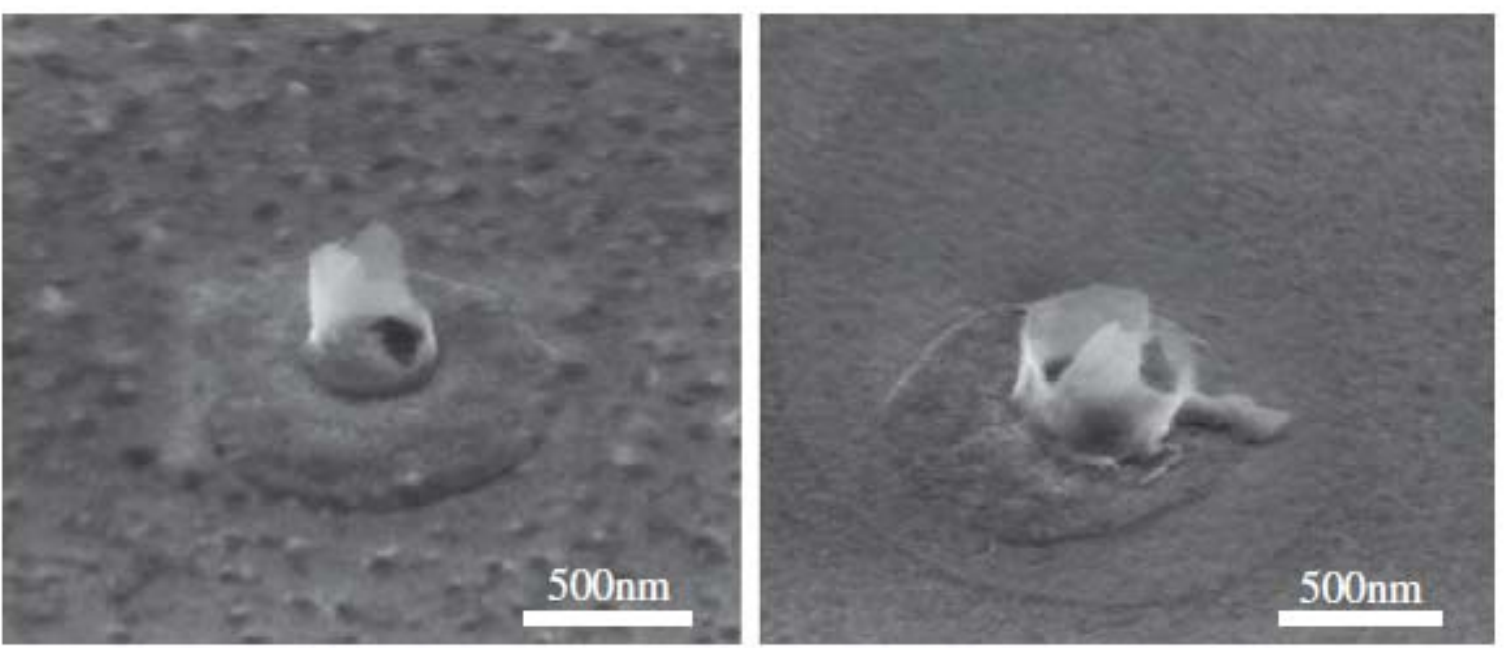

Fig. 12. Failed hollow indium pillars. 